\title{
Growth and characterization of pyrene crystals on carbon nanofibers
}

Satoshi Kubota, ${ }^{\mathrm{a}}$ Hiromasa Nishikiori, ${ }^{\mathrm{b}}$ Nobuaki Tanaka, ${ }^{\mathrm{a}, \mathrm{b}}$ Morinobu Endo, ${ }^{\mathrm{c}, \mathrm{d}}$ Tsuneo Fujii ${ }^{\mathrm{a}}$ $b^{*}$

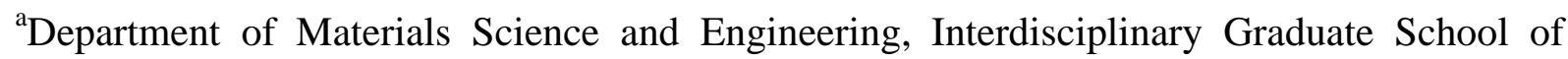
Science and Technology, Shinshu University, Wakasato, Nagano 380-8553, Japan

${ }^{\mathrm{b}}$ Department of Environmental Science and Technology, Faculty of Engineering, Shinshu University, Wakasato, Nagano 380-8553, Japan

${ }^{\mathrm{c}}$ Department of Mathematics and System Development, Interdisciplinary Graduate School of Science and Technology, Shinshu University, Wakasato, Nagano 380-8553, Japan

${ }^{\mathrm{d}}$ Institute of Carbon Science and Technology, Shinshu University, Wakasato, Nagano 380-8553, Japan

\section{Abstract}

Pyrene crystals were grown on carbon nanofibers (CNFs) by dispersing pyrene polycrystals and CNFs in water during ultrasonic irradiation, and they were characterized by scanning electron microscopy, X-ray diffractometry (XRD) and spectroscopy. The XRD measurements indicated that the orientation and size of the pyrene crystals on the CNF aggregates were different from that of the added pyrene polycrystals. Based on the 
spectroscopic properties of the pyrene crystals on the CNFs, the pyrene crystals on the CNF aggregates and on the individual CNFs were determined to be polycrystals and single crystals, respectively. These results indicate that pyrene crystals are produced on the CNFs by recrystallization of the added pyrene polycrystals and their crystal states depend on the aggregation state of the CNFs.

Keywords: Carbon nanofibers; Pyrene; Single crystals; Absorption; Fluorescence

*Corresponding author: Tsuneo Fujii

Phone: +81-26-269-5535

Fax: $\quad+81-26-269-5550$

E-mail address: tsfujii@shinshu-u.ac.jp 


\section{Introduction}

Nanocarbons, such as carbon nanotubes (CNTs) [1,2] and carbon nanofibers (CNFs), [3-5] are promising materials for use in various mechanical, electronic and optical devices because of their remarkable mechanical and unique electronic properties [6-11]. The preparation of the CNFs is technologically easier and economically more favorable for practical applications compared to that of the CNTs. The CNFs bonded to organic molecules are available for optoelectronic devices utilizing the excited-state energy transfer or electron transfer from the tethered molecules to the nanocarbons [12-14]. In addition, noncovalent modifications of the CNFs using aromatic molecules through a $\pi-\pi$ interaction have also been utilized [15-19]. Instead of an aromatic molecular modification, the deposition of aromatic crystals on the CNF surfaces enhances two properties of the devices; one is an increase in the absorption efficiency of the aromatic molecules in the crystal state on the CNFs due to higher density of the packed molecules, and the other is a shift to longer wavelengths and an extension of the absorption range due to the formation of the aromatic crystal bands. However, in the case of the crystal modification on the CNFs, the crystal size affects the energy transfer efficiency due to the confined exciton migration range. Therefore, the formation of a smaller crystal on the CNFs is required for a higher energy transfer efficiency. 
We have previously shown that pyrene molecules are adsorbed onto the CNFs through

a $\pi-\pi$ interaction by observing the fluorescence spectra of pyrene on the CNFs [19]. During this procedure, pyrene molecules were adsorbed on the CNFs dispersed by ultrasonic irradiation in a mixed water/ethanol solution of pyrene. To enhance the dispersibility of the CNFs in solution, some functional groups were introduced on the surface of the CNFs by acid treatment. The acid treatment of the CNFs with nitric acid and sulfuric acid leads to oxidized groups such as $-\mathrm{COOH}$ and $-\mathrm{C}=\mathrm{O}$ in the graphene sheet of the materials $[18,21-23]$.

In this study, we tried to prepare the pyrene crystals on the acid-treated CNFs by adding a considerable amount of pyrene that exceeded the solubility and the acid-treated CNFs in water and ultrasonically agitating. The pyrene molecules can be easily adsorbed on the CNFs dispersed by ultrasonic irradiation in water due to their hydrophobic characteristics. Furthermore, the ultrasonication can create a supersaturated solution of pyrene near the CNFs by rapid local cooling after an increase in the local temperature created by bubble collapse and concomitant release of shock waves [24]. Therefore, it is expected that the heterogeneous nucleation and the crystallization of pyrene on the CNFs are attained by agitating the starting material of the pyrene and the acid-treated CNFs in only water during ultrasonic irradiation. Characterization of the pyrene on the CNFs was carried out by scanning electron microscopy (SEM), X-ray diffractometry (XRD) and spectroscopy. 


\section{Experimental}

The pyrene (Wako Pure Chemicals, S grade) was recrystallized three times from ethanol. It is referred to as the original pyrene polycrystals in this report. The CNFs (VGCF-G) having a diameter of ca. $200 \mathrm{~nm}$, a length of ca. 10 - $20 \mu \mathrm{m}$, and a surface area of ca. $15 \mathrm{~m}^{2} \mathrm{~g}^{-1}$ were provided by Showa Denko Co., Ltd. The cutting and functionalization of the CNFs was carried out using a mixture of concentrated nitric acid and sulfuric acid, as previously reported [18-21]. The acid-treated CNF sample was designated AA-CNF in this report.

The AA-CNF (0.50 mg) and the original pyrene crystals $(10 \mathrm{mg})$ were added to water (10 ml) and ultrasonically agitated. The crystallization of pyrene on the CNFs is expected to be attained by this procedure. Therefore, the resultant suspension is expected to contain individual CNFs loading pyrene crystals, CNF aggregates loading pyrene crystals, pyrene molecules and added pyrene crystals.

The deposition of the pyrene crystals on the CNF aggregates and on the individual CNFs was confirmed by SEM (Hitachi S-4100) and XRD (Rigaku Rint 2200V). The preparation procedures of the samples for SEM observation and XRD measurement are shown in Scheme 1. The two different states of the CNF aggregates were separated from the suspension by filtration. The aqueous suspension containing the added pyrene polycrystals and AA-CNF ultrasonically agitated for 15 days was filtered through a coarse 
filter paper having $5.0 \mu \mathrm{m}$ pores to separate the larger aggregates of the added pyrene polycrystals and AA-CNF. The separated substance was designated Sample A1. The resultant filtrate was then filtered through a finespun filter paper having $0.2 \mu \mathrm{m}$ pores to separate the smaller CNF aggregates. The separated substance was designated Sample A2.

(Scheme 1)

The following two reference samples were prepared in order to investigate whether or not the crystallization of the pyrene on the CNF aggregates occurred by ultrasonic irradiation. The AA-CNF (0.50 mg) and the original pyrene crystals $(10 \mathrm{mg})$ were then added to water (10 ml). The solutions were left or stirred at $313 \mathrm{~K}$ for 15 days without ultrasonication. This temperature was the same as in the ultrasonication bath. Since almost all of the AA-CNF and the pyrene crystals in the system were aggregated, they were separated by a coarse filter paper having $5.0 \mu \mathrm{m}$ pores. The separated substances from the suspensions not stirred or stirred were designated Sample CA1 and Sample CA2, respectively.

The individual CNFs were separated from the suspension as follows. The supernatant was obtained by centrifugation of the suspension at 4,000 g. Since the centrifugation cannot spin down the individual CNFs, the individual CNFs loading pyrene crystals and pyrene molecules are expected to be contained in the supernatant. The supernatant was then dried and this specimen was designated Sample B. 
The following reference sample was prepared in order to investigate whether the crystallization of pyrene on the individual CNFs occurred during the solvent evaporation. The AA-CNF $(0.50 \mathrm{mg})$ was dispersed in water $(10 \mathrm{ml})$ by ultrasonic irradiation. They were centrifuged in order to leave only the individual CNFs in the supernatant. The, excess pyrene was then added to this supernatant. After saturation of the pyrene at $313 \mathrm{~K}$ was attained, the suspension was filtered though a finespun filter paper having $0.2 \mu \mathrm{m}$ pores in order to remove the undissolved pyrene crystal. The resultant filtrate was dried and this specimen was designated Sample CB.

The UV absorption (Shimadzu UV-3150 spectrophotometer), fluorescence and fluorescence excitation spectra (Shimadzu RF-5300 spectrofluorophotometer) of the pyrene on the CNFs were measured. The aim of these analyses was to investigate the pyrene crystal states on the CNF aggregates or on the individual CNFs. The preparation procedures of the samples for the spectroscopy are shown in Scheme 2. For the pyrene crystals on the individual CNFs, the spectral measurements were done for the supernatant obtained by centrifuging the suspensions containing the added pyrene polycrystals and AA-CNF ultrasonically agitated for 15 days. This supernatant was designated Supernatant A.

(Scheme 2) 
Furthermore, the absorption spectra of the following three reference supernatants were measured. The first sample was prepared as follows in order to investigate whether or not the heterogeneous nucleation of the pyrene on the AA-CNF occurred. The supernatant obtained by centrifuging the suspension of the pyrene polycrystals without the AA-CNF ultrasonically agitated for 15 days. This supernatant was designated Supernatant CA1. The second sample was prepared as follows in order to investigate whether or not the crystallization occurred by stirring. The supernatant was obtained by centrifuging the suspensions containing the added pyrene polycrystals and AA-CNF stirred for 15 days at 313 K. This supernatant was designated Supernatant CA2. The third sample was prepared as follows in order to investigate whether or not the crystallization in the AA-CNF dispersed water occurred by stirring. The AA-CNF $(1.0 \mathrm{mg})$ was dispersed in water $(20 \mathrm{ml})$ by ultrasonic irradiation, and then it was centrifuged to leave only the individual CNFs in the supernatant. Pyrene $(10 \mathrm{mg})$ was added to this supernatant $(10 \mathrm{ml})$. The solution was stirred for 15 days at $313 \mathrm{~K}$. Finally, the supernatant was obtained by centrifugation of the stirred solution. This supernatant was designated Supernatant CA3.

\section{Results and discussion}

\subsection{Confirmation of deposition of pyrene crystals on carbon nanofibers}


Figure 1 shows the SEM images of Samples CA1, CA2, A1, A2, CB and B. In the image of Sample CA1 shown in Fig.1 (a), the AA-CNF does not seem to be covered by the pyrene crystals. The diameter of the AA-CNF in Sample CA1 is not different from that of the added AA-CNF. $\quad$ On the other hand, the AA-CNF in Sample CA2 shown in Fig.1 (b) has a larger diameter compared to that of Sample CA1. Thus the pyrene crystals are attached to the AA-CNF. In the images of Samples A1 and A2 shown in Fig. 1 (c) and (d), respectively, the tubular shape of the CNFs is not clearly observed because a large amount of the pyrene crystals surround the AA-CNF. The AA-CNF in Sample A1 seems to be surrounded by more pyrene crystals than that in Sample A2. These results suggest that the pyrene crystals exist on the AA-CNF aggregates by stirring or ultrasonication.

Figures 1 (e) and (f) show Samples CB and B, respectively. These samples seem to be the individual CNFs on the based on the high magnification SEM images. The images of Samples CB and B displayed microasperities on the AA-CNF. These asperities are predicted to be pyrene nanocrystals on the AA-CNF. From the SEM images, it was not clear that the crystallization of pyrene occurred or not by ultrasonication because the AA-CNF in both samples have microasperities. Therefore, we will try to confirm the crystallization by spectroscopic measurements in $\S 3.2$.

(Figure 1) 
Figure 2 shows the XRD patterns of the AA-CNF and Samples CA1, CA2, A1 and A2.

Although we have observed the XRD patterns of Samples CB and B, we could not obtain a significant signal due to low density of the CNFs in these samples. In the XRD patterns for Samples CA1, CA2, A1 and A2, the peak positions of the pyrene crystals were identical. Since each characteristic interplane spacing of the pyrene crystal was unchanged in Samples CA1, CA2, A1 and A2, they have the same crystal structure.

The pyrene crystal belongs to the sandwich-herringbone class; two parallel molecules are arranged in a sandwich motif through $\pi-\pi$ interactions, and each motif is arranged in a herringbone fashion favoring $\mathrm{C}-\mathrm{H} \cdots \pi$ interactions[25]. The relative intensity of the peaks for the pyrene crystals in Samples CA1, CA2, A1 and A2 differ from each other. We focused our discussion on the $\left(\begin{array}{lll}0 & 0 & 1\end{array}\right)$ plane of the pyrene crystal. The relative intensities of the $\left(\begin{array}{lll}0 & 0 & 1\end{array}\right)$ plane of the pyrene crystal for Samples CA2, A1 and A2 are lower than that for Sample CA1. The order of the relative intensity of the $\left(\begin{array}{lll}0 & 0 & 1\end{array}\right)$ plane for Samples CA2, A1 and A2 was observed as Sample A2 < Sample CA2 < Sample A1. The relative intensities of the peak at $26.6^{\circ}$ assigned to the AA-CNF for Samples CA2, A1and A2 show the order of Sample A1 < Sample CA2 < Sample A2. Therefore, the relative intensities of the $\left(\begin{array}{lll}0 & 0 & 1\end{array}\right)$ plane decreased with an increase in the relevant amount of the AA-CNF. This means that a relatively small amount of pyrene molecules was aligned in the c-axis direction on the AA-CNF. 
The full width at half maximum (FWHM) of the diffraction peak for the pyrene crystals in Samples CA2 and A1 is almost identical to that of Sample CA1. On the other hand, that of Sample A2 differs from that of Sample CA1. Based on the Scherrer formula, the crystallite sizes of the pyrene in the sample were estimated. For estimation using $2 \theta$ $=10.8^{\circ}$, which corresponds to the $\left(\begin{array}{lll}0 & 0 & 1\end{array}\right)$ plane, the crystallite sizes of the pyrene in the direction in Samples CA1, CA2, A1 cannot be evaluated due to their small FWHM. It is inferred that these are more than $200 \mathrm{~nm}$. On the other hand, that in Sample A2 was evaluated to be $100 \mathrm{~nm}$. For estimation using $2 \theta=23.6^{\circ}$, which corresponds to the (2 2 0) plane, the crystallite sizes of the pyrene in the direction in Samples CA1, CA2, A1 and A2 were evaluated to be $80 \mathrm{~nm}, 80 \mathrm{~nm}, 80 \mathrm{~nm}$ and $60 \mathrm{~nm}$, respectively. The crystallite sizes of the pyrene in Sample A2 were smaller than that of Sample CA1. Also, the difference between the crystallite sizes of the (llllll) and (2 $\left.\begin{array}{lll}2 & 2 & 0\end{array}\right)$ directions of pyrene in Sample A2 was smaller than that for Sample CA1. This means that a relative amount of pyrene molecules aligned in the c-axis in the pyrene crystallite in Sample A2 was smaller compared to that of Sample CA1. These results indicate smaller and relatively isotopic pyrene crystals were grown on the AA-CNF in Sample A2.

(Figure 2)

3.2. Identification of pyrene crystal states on carbon nanofibers by spectroscopic measurements 
The absorption spectra of the pyrene crystals on the AA-CNF were measured in order to understand the pyrene crystal states on the AA-CNF. Figure 3 shows the UV absorption spectra of the pyrene on the CNF aggregates and on the individual CNFs. Fig. 3(a) shows the absorption spectra of the pyrene molecules in water, the original pyrene polycrystals, and Sample A2. The absorption spectrum of the original pyrene polycrystals exhibits bands with peaks at 322, 338, and ca. $376 \mathrm{~nm}$ in the $300-400 \mathrm{~nm}$ region. The former two peaks are assigned to the transitions from the ground state to the ${ }^{1} \mathrm{~L}_{\mathrm{a}}$ exciton states. These peaks shifted to the lower energy side compared to that of the pyrene molecules in water. This shift is attributed to the interaction on the dimeric structure in the pyrene crystal [26]. The peak at ca. $376 \mathrm{~nm}$ is attributed to the transition from the ground state to the bottom of the free exciton state, the ${ }^{1} \mathrm{~L}_{b}$ exciton state, in the pyrene polycrystal [27-29]. The absorption spectra of Sample A2 almost corresponds to that of the original pyrene polycrystals, whereas the XRD patterns of these samples are different from that of the added pyrene polycrystals. This indicates that the orientation and size of the resultant crystals on the CNF aggregates is different from that of the added pyrene polycrystals, and the crystal state remained as polycrystals.

Figure 3(b) shows the absorption spectra of Supernatants A, CA1, CA2, and CA3. The absorption spectrum of Supernatant A exhibits three bands with peaks at 320, 336, and $353 \mathrm{~nm}$. This absorption profile revealed a significantly different shape from that of the 
original pyrene polycrystals. The absorption spectrum of Supernatant A is similar to that of the pyrene single crystals exhibiting three sharp peaks at ca. 320, 338, and $355 \mathrm{~nm}$ as reported by Takahashi et al. [30]. The spectrum of the pyrene single crystals is shifted to the short wavelength side compared to that of the original pyrene polycrystals. This shift is attributed to the formation of a broader band in the polycrystals compared to the single crystals due to perturbation between the crystals grain boundaries.

On the other hand, the spectrum of Supernatant CA1 indicated the same spectrum as that of the pyrene molecules in water instead of the pyrene crystal band. The crystallization of the pyrene single crystals did not occur in the absence of the AA-CNF. The spectrum of Supernatant CA2 is similar to that of Supernatant CA1. Since the absorption of the AA-CNF did not appear in the spectrum of Supernatant CA2, the AA-CNF was not dispersed in water by stirring. Consequently, the single pyrene crystals were not produced. The spectrum of Supernatant CA3 indicated the peaks of pyrene molecules in water on the background due to absorption of the AA-CNF. In spite of the presence of the AA-CNF, the crystallization of pyrene did not occur by stirring. These results mean that the heterogeneous nucleation and crystal growth of the pyrene single crystals on the AA-CNF dispersed by ultrasonication are attained from the pyrene supersaturated solution created by ultrasonication.

(Figure 3) 
In order to investigate in more detail the state of the pyrene on the individual AA-CNF in Supernatant A, the fluorescence and fluorescence excitation spectra were measured. Figure 4 shows the fluorescence spectra of the original pyrene polycrystals and Supernatant A. All of the fluorescence spectra were corrected by subtracting the scattering of incident light. The fluorescence spectra of the original pyrene polycrystals and Supernatant A excited at 360 nm show similar spectral features and exhibit only one peak at $460 \mathrm{~nm}$. The peak at 460 $\mathrm{nm}$ corresponds to the fluorescence from the excimer-like state induced by local self-trapping of the excitation energy and is related to a specific dimeric interaction in the crystal lattice [27-29,31]. Although the free exciton fluorescence is generally observed at $376 \mathrm{~nm}$ in the pyrene polycrystals, it was apparently not observed here. This suggested that the free exciton fluorescence was concealed behind the intense scattering of light. The fluorescence spectrum of Supernatant A excited at $335 \mathrm{~nm}$ exhibits a dual fluorescence. One is the vibronic bands seen at $372-392 \mathrm{~nm}$ and is assigned to the pyrene monomer in water $[26,32,33]$. The other is the broad band, which has a peak at $460 \mathrm{~nm}$, due to an excimer-like fluorescence, as observed in the spectrum at the $360 \mathrm{~nm}$ excitation. These results indicate that the absorption band at ca. $360 \mathrm{~nm}$ is attributed to only the pyrene crystals, and that at ca. $335 \mathrm{~nm}$ is attributed to not only the pyrene crystals, but also the pyrene molecules in water. Furthermore, according to the report by Takahashi et al., the fluorescence of the pyrene single crystals indicates the same excimer-like fluorescence as that of the original pyrene 
polycrystals [34]. Therefore, the fluorescence having a peak at $460 \mathrm{~nm}$ in Supernatant A will be attributed to the pyrene single crystals. We will try to confirm this by measurement of fluorescence excitation spectrum.

\section{(Figure 4)}

Figure 5 shows the fluorescence excitation spectra of the pyrene molecules and crystals under various conditions. The fluorescence excitation spectra of Supernatant A depend on the observed wavelength. The spectrum of Supernatant A monitored at $390 \mathrm{~nm}$ exhibits peaks at 307, 320 and $335 \mathrm{~nm}$. These peaks correspond to the transition to the ${ }^{1} \mathrm{~L}_{\mathrm{a}}$ molecular state of the pyrene in solution [26]. On the other hand, the spectrum of Supernatant A monitored at $480 \mathrm{~nm}$ exhibits peaks at 340, 360 and $376 \mathrm{~nm}$. There are two obvious differences between the spectra of Supernatant A monitored at $480 \mathrm{~nm}$ and the original pyrene polycrystals having peaks at 343, 367 and $376 \mathrm{~nm}$. One is the blue shift of the $360 \mathrm{~nm}$ peak of the pyrene crystals in Supernatant A compared to the peak at $367 \mathrm{~nm}$ of the original pyrene polycrystals. As already mentioned about the absorption spectra, this blue shift is attributed to the pyrene single crystals growing on the CNF surface. However, there is a red shift in the peak around $360 \mathrm{~nm}$ in the excitation spectrum compared to that in the UV absorption spectrum. The absorption peak shift to the longer wavelength side accompanied by an increase in the crystal size in the perylene crystal has been reported [35]. Therefore, the red shift suggests that the fluorescence efficiently originates from the larger 
crystals having an absorption peak on the longer wavelength side. The other fact is the relative intensity of the $376 \mathrm{~nm}$ peak of the pyrene crystals in Supernatant A which is lower than that of the original pyrene polycrystals. These results suggest that the fluorescence at $460 \mathrm{~nm}$ in Supernatant A was mostly generated from the pyrene single crystals and a small amount from the pyrene polycrystals. The free exciton band in the pyrene polycrystals at $376 \mathrm{~nm}$ that appeared in the fluorescence excitation spectrum of Supernatant A did not distinctly appear in the absorption spectrum. This suggests that the fluorescence efficiency of the pyrene single crystals on the AA-CNF is lower than that of the pyrene polycrystals on the AA-CNF. Therefore, this result implies that the energy transfer originating from the pyrene single crystals to the CNFs is more efficient than that from the pyrene polycrystals due to enhancement of the interaction between the smaller pyrene single crystal and the AA-CNF. (Figure 5)

\section{Conclusions}

We confirmed the growth of pyrene crystals on the CNFs by dispersing the original pyrene polycrystals and the CNFs in water during ultrasonication. Based on the XRD analysis, it was determined that the orientation and size of the pyrene crystals on the CNFs is different from that of the added pyrene polycrystals. This suggests that the crystallization of the pyrene on the CNF aggregates occurred. 
From the spectroscopic measurements, it was determined that the pyrene crystals grew as single crystals on the individual CNFs and as polycrystals on the CNF aggregates. On the surface of the individual CNFs dispersed by ultrasonication, the nucleation occurs from the pyrene supersaturated solution created by ultrasonication. Consequently, the pyrene single crystals are formed on the AA-CNF. The growth mechanism of pyrene crystals on the CNF aggregates was suggested as follows. The single pyrene crystals grew during the initial stage. They then gradually became larger polycrystals due to the closer spacing of the CNFs to each other. Therefore, the single crystals on the individual CNFs have a smaller crystal size compared to the polycrystals on the CNF aggregates.

\section{Acknowledgements}

This research was supported by CLUSTER (the second stage) and Grant-in-Aids for Scientific Research (No. 20915012) from the Ministry of Education, Culture, Sportst Science and Technology, Japan.

\section{References}

[1] S. Iijima, Nature 354 (1991) 56.

[2] S. Iijima, T. Ichihashi, Nature 363 (1993) 603. 
[3] A. Oberlin, M. Endo, T. Koyama, J. Cryst. Growth. 32 (1976) 335.

[4] M. Endo, Chemtech. 18 (1988) 568.

[5] M. Endo, Y. A. Kim, T. Hayashi, K. Nishimura, T. Matusita, K. Miyashita, M. S. Dresselhaus, Carbon 39 (2001) 1287.

[6] R. H. Baughman, A. A. Zakhidov, W. A. de Heer, Science 297 (2002) 787.

[7] R. Singh, D. Pantarotto, D. McCarthy, O. Chaloin, J. Hoebeke, C. D. Partidos, J-P. Briand, M. Prato, A. Bianco, K. Kostarelos, J. Am. Chem. Soc. 127 (2005) 4388.

[8] J. S. Tans, R. M. A. Verschueren, C. Dekker, Nature 393 (1998) 49.

[9] R. Saito, G. Dresselhaus, M.S. Dresselhaus, Phys. Rev. B 61 (2000) 2981.

[10] M. J. O’Connell, S. M. Bachilo, C. B. Huffman, V. C. Moore, M. S. Strano, E. H. Haroz, K. L. Rialon, P. J. Boul, W. H. Noon, C. Kittrell, J. Ma, R. H. Hauge, R. B. Weisman, R. E. Smalley, Science 297 (2002) 593.

[11] Y. Maniwa, K. Matsuda, H. Kyakuno, S. Ogasawara, T. Hibi, H. Kadowaki, S. Suzuki, Y. Achiba, H. Kataura, Nature materials 6 (2007) 135.

[12] L. Qu, B. R. Martin, W. Huang, K. Fu, D. Zweifel, Y. Lin, J. Chem. Phys. 117 (2002) 8089. 
[13] H. Li, E. M. Kose, L. Qu, Y. Lin, B. R. Martin, B. Zhou, A. B. Harruff, F. L. Allard, Y. Sun, J. Photochem. Photobio. A 185 (2007) 94.

[14] M. Álvaro, P. Atienzar, L. J. Bourdelande, H. García, Chem. Phys. Lett. 384 (2004) 119.

[15] J. R. Chen, Y. Zhang, D. Wang, H. Dai, J. Am.Chem. Soc. 123 (2001) 3838.

[16] H. Nishikiori, N. Tanaka, S. Kubota, M. Endo, T. Fujii, Chem. Phys. Lett. 390 (2004) 389.

[17] S. Kubota, H. Nishikiori, N. Tanaka, M. Endo, T. Fujii, Chem. Phys. Lett. 412 (2005) 223.

[18] S. Kubota, H. Nishikiori, N. Tanaka, M. Endo, T. Fujii, J. Phys. Chem. B 109 (2005) 23170.

[19] T. Tanigaki, H. Nishikiori, S. Kubota, N. Tanaka, M. Endo, T. Fujii, Chem. Phys. Lett. 448 (2007) 218.

[20] Nishikiori, H.; Tanaka, N.; Tanigaki, T.; Endo, M.; Fujii, T. J. Photochem. Photobio. A 193 (2008) 161. 
[21] J.; Liu, A. G. Rinzler, H. Dai, J. H. Hafner, R. K. Bradley, P. J. Boul, A. Lu, T. Iverson, K. Shelimov, C. B. Huffman, F. R. Macias, Y. S. Shon, T. R. Lee, D. T. Colbert, R. E. Smallery, Science 280 (1998) 1253.

[22] P. V. Lakshminarayanan, H. Toghiani, C. U. Pittmann Jr., Carbon 42 (2004) 2433.

[23] M. A. Hamon, H. Hui, P. Bhowmik, H. M. E. Itkis, R. C. Haddon, Appl. Phys. A 74 (2002) 333.

[24] M. D. Luque de Castro, F. Priego-Capote, Ultra. Sonochem. 14 (2007) 717.

[25] F. P. A. Fabbiani, D. R. Allam, S. Parsons, C. R. Pulham, Acta Cryst. B62 (2006) 826.

[26] T. Fujii, E. Shimizu, S. Suzuki, J.Chem. Soc. Faraday Trans.1 84 (1988) 4387.

[27] A. Matsui, H. Nishimura, J. Phys. Soc. Jpn. 49 (1980) 657.

[28] T. Nakanishi, K. Mizuno, A. Matsui, H. Nishimura, J. Luminescence 47 (1991) 303.

[29] A. Matsui, T. Nakamura, S. Nakatani, T. Ohno, K. Mizuno, Syntheic Metals 64 (1994) 177.

[30] N. Takahashi, B Gombojav, T. Yoshinari, S. Nagasaka, Y. Takahashi, A. Yamamoto, T. Goto, A. Kasuya, J. Sol. Sta. Chem. 177 (2004) 3479. 
[31] R. Seyfang, H. Port, P. Fischer, H. C. Wolf, J. luminescence 51 (1992) 197.

[32] T. Fujii, K. Murayama, N. Negishi, M. Anpo, E. J. Winder, D. R. Neu, A. B. Ellis, Bull. Chem. Soc. Jpn. 66 (1993) 739.

[33] A. Nakajima, Bull. Chem. Soc. Jpn. 44 (1971) 3272.

[34] N. Takahashi, B Gombojav, S.Yoshinari, Y. Takahashi, T. Nagasaka, A. Yamamoto, T. Goto, A. Kasuya, J. Phys. Soc. Jpn. 76 (2007) 034703.

[35] H. Kasai, H. Oikawa, S. Okada, H. Nakanishi, Bull. Chem. Soc. Jpn. 71 (1998) 2597. 


\section{Figure captions}

\section{Scheme 1}

The preparation procedures of (a) ultrasonically agitated samples and (b) control samples for

SEM observation and XRD measurement

\section{Scheme 2}

The preparation procedures of (a) ultrasonically agitated samples and (b) control samples for spectroscopy

\section{Figure 1}

SEM images of (a) Sample CA1, (b) Sample CA2, (c) Sample A1, (d) Sample A2, (e) Sample CB, and (f) Sample B.

\section{Figure 2}

XRD patterns of (1) the AA-CNF, (2) Sample CA1, (3) Sample CA2, (4) Sample A1, and (5) Sample A2.

\section{Figure 3}

(a) UV absorption spectra of (1) pyrene molecules in water (saturated solution), (2) the original pyrene polycrystals and (3) Sample A2. 
(b) UV absorption spectra of (1) Supernatant A, (2) Supernatant CA1, (3) Supernatant CA2, and (4) Supernatant CA3

\section{Figure 4}

Fluorescence spectra of (1) the original pyrene polycrystals and (2, 3) Supernatant A. (1) and (2) were excited at $360 \mathrm{~nm}$. (3) was excited at $335 \mathrm{~nm}$.

\section{Figure 5}

Fluorescence excitation spectra of (1) pyrene molecules in water (saturated solution) (2) the original pyrene polycrystals and $(3,4)$ Supernatant A. (1) and (3) were monitored at 390 nm. (2) and (4) were monitored at $480 \mathrm{~nm}$. 
(a)

The AA-CNF (0.50 mg) and the original pyrene crystals $(10 \mathrm{mg})$ were added to water $(10 \mathrm{ml})$ and ultrasonically agitated for 15 days

Filtration with a coarse filter paper having $5.0 \mu \mathrm{m} \downarrow$

\section{Sample A1}

Filtration with a finespun filter paper having $0.2 \mu \mathrm{m}$ pores

\section{Sample A2}

Centrifugation at 4,000 $\mathrm{g}$
Drying

\section{Sample B}

(b)

\section{Comparison with Samples A1 and A2}

The AA-CNF (0.50 mg) and the original pyrene crystals $(10 \mathrm{mg})$ were added to water $(10 \mathrm{ml})$

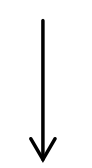

Keeping at $313 \mathrm{~K}$ Keeping at $313 \mathrm{~K}$ for 15 days for 15 days with without stirring stirring

$$
\downarrow \begin{aligned}
& \text { Filtration with a } \\
& \text { coarse filter paper } \\
& \text { having } 5.0 \mu \mathrm{m} \\
& \text { pores }
\end{aligned}
$$

\section{Sample CA1}

\section{Sample CA2}

\section{Comparison with Sample B}

The AA-CNF $(0.50 \mathrm{mg})$ was
dispersed in water $(10 \mathrm{ml})$ by
ultrasonic irradiation.

Centrifugation at $4,000 \mathrm{~g}$

Supernatant containing individual CNFs

Adding excess pyrene to attain saturation at $313 \mathrm{~K}$.

Filtration with a finespun filter paper having $0.2 \mu \mathrm{m}$ pores.

Filtrate Drying

\section{Sample CB}


(a)

The AA-CNF (0.50 mg) and the original pyrene crystals $(10 \mathrm{mg})$ were added to water $(10 \mathrm{ml})$ and ultrasonically agitated for 15 days

Centrifugation

at $4,000 \mathrm{~g}$

\section{Supernatant A}

(b)

\section{Comparison with Supernatant A}

The original pyrene crystals (10 mg) were added to water $(10 \mathrm{ml})$ and ultrasonically agitated for 15 days
The AA-CNF (1.0 mg) was dispersed in water $(20 \mathrm{ml})$ by ultrasonic irradiation.

$$
\downarrow \begin{aligned}
& \text { Centrifugation } \\
& \text { at } 4,000 \mathrm{~g}
\end{aligned}
$$

Supernatant CA1
Supernatant containing individual CNFs (10 ml)
The AA-CNF (0.50 mg) and the original pyrene crystals (10 mg) were added to water $(10 \mathrm{ml})$ and stirred for 15 days at $313 \mathrm{~K}$

Centrifugation at $4,000 \mathrm{~g}$

\section{Supernatant CA2}

Centrifugation at 4,000 g 
(a)

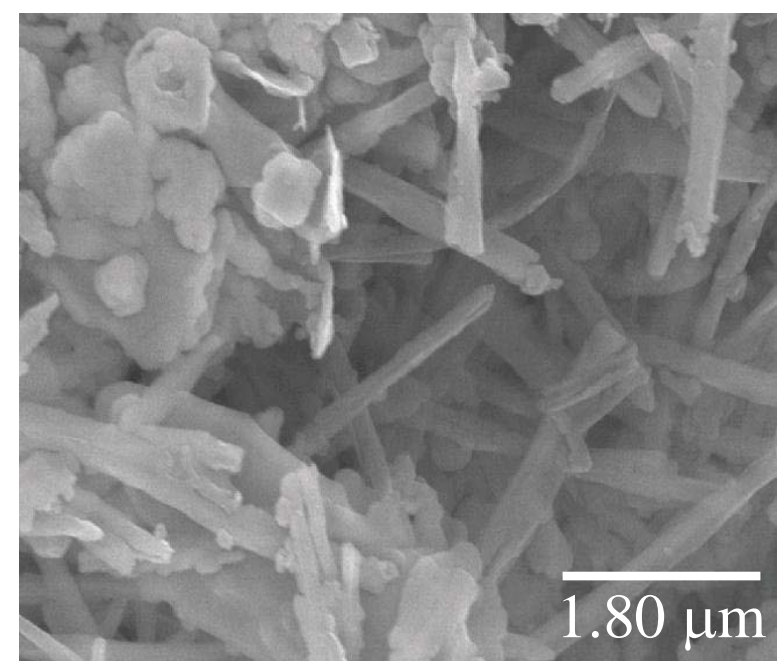

(c)

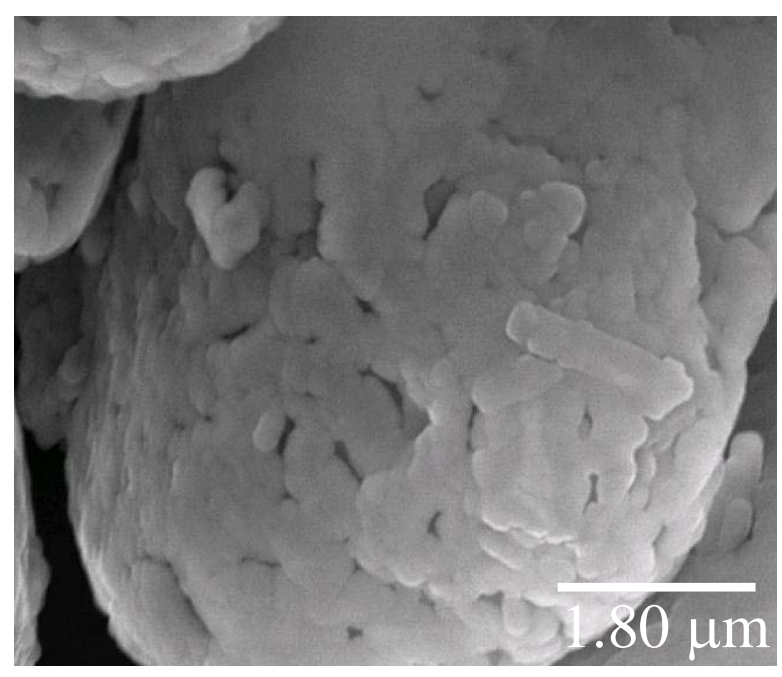

(e)

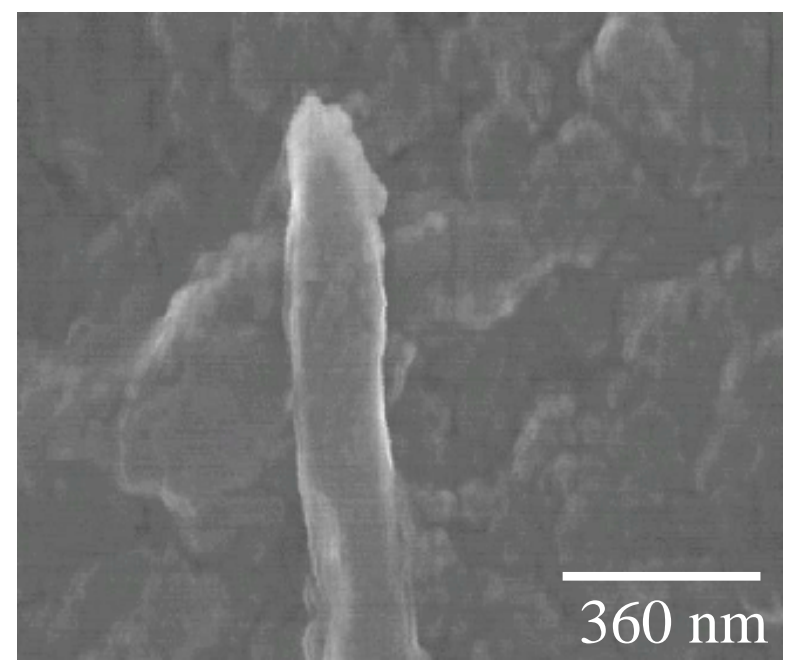

(b)

Figure 1

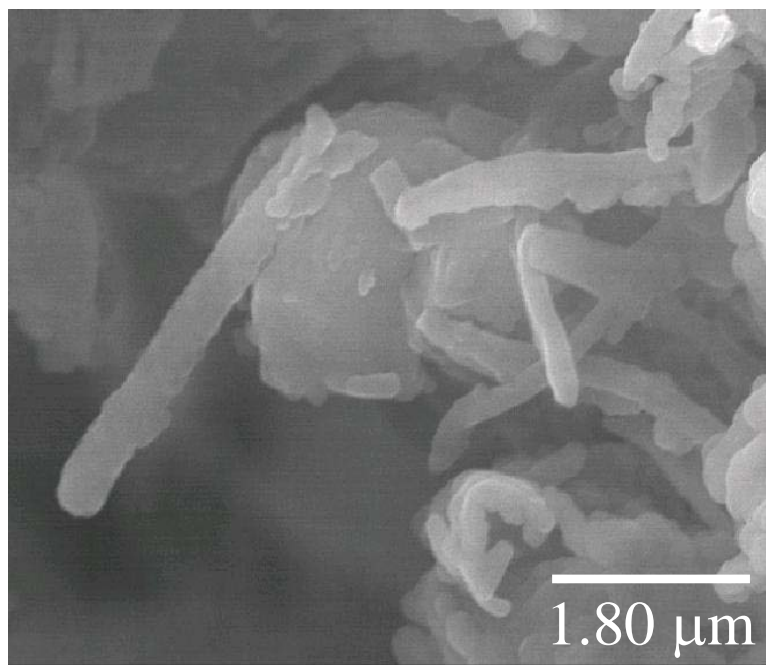

(d)

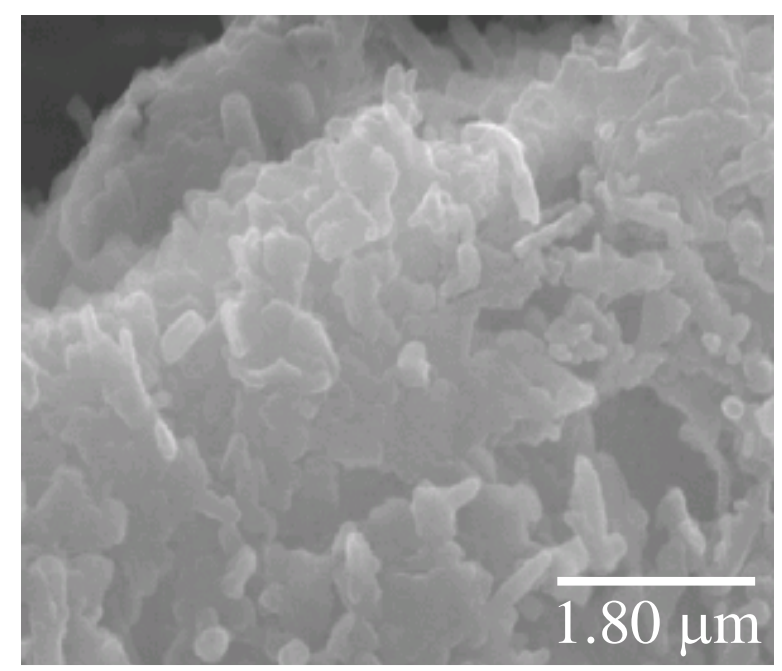

(f)

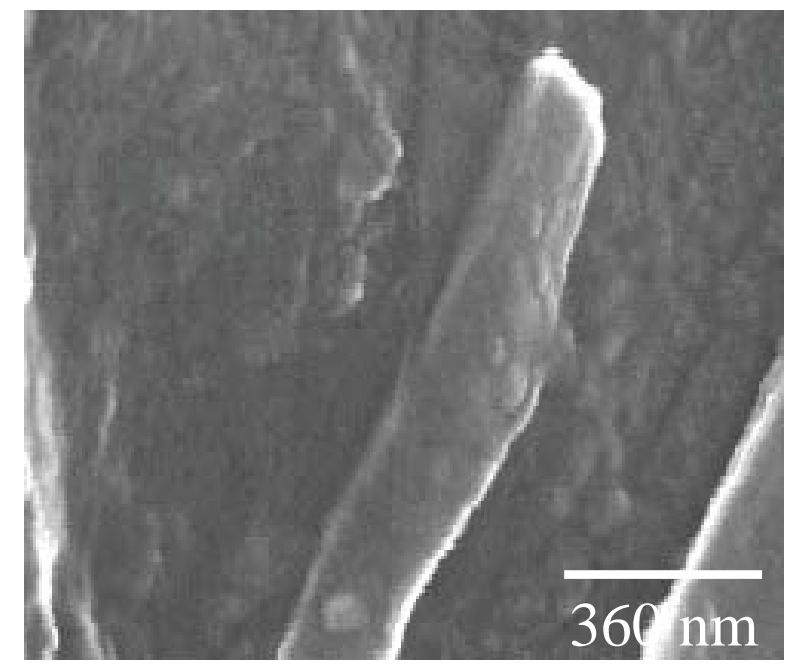


Figure 2

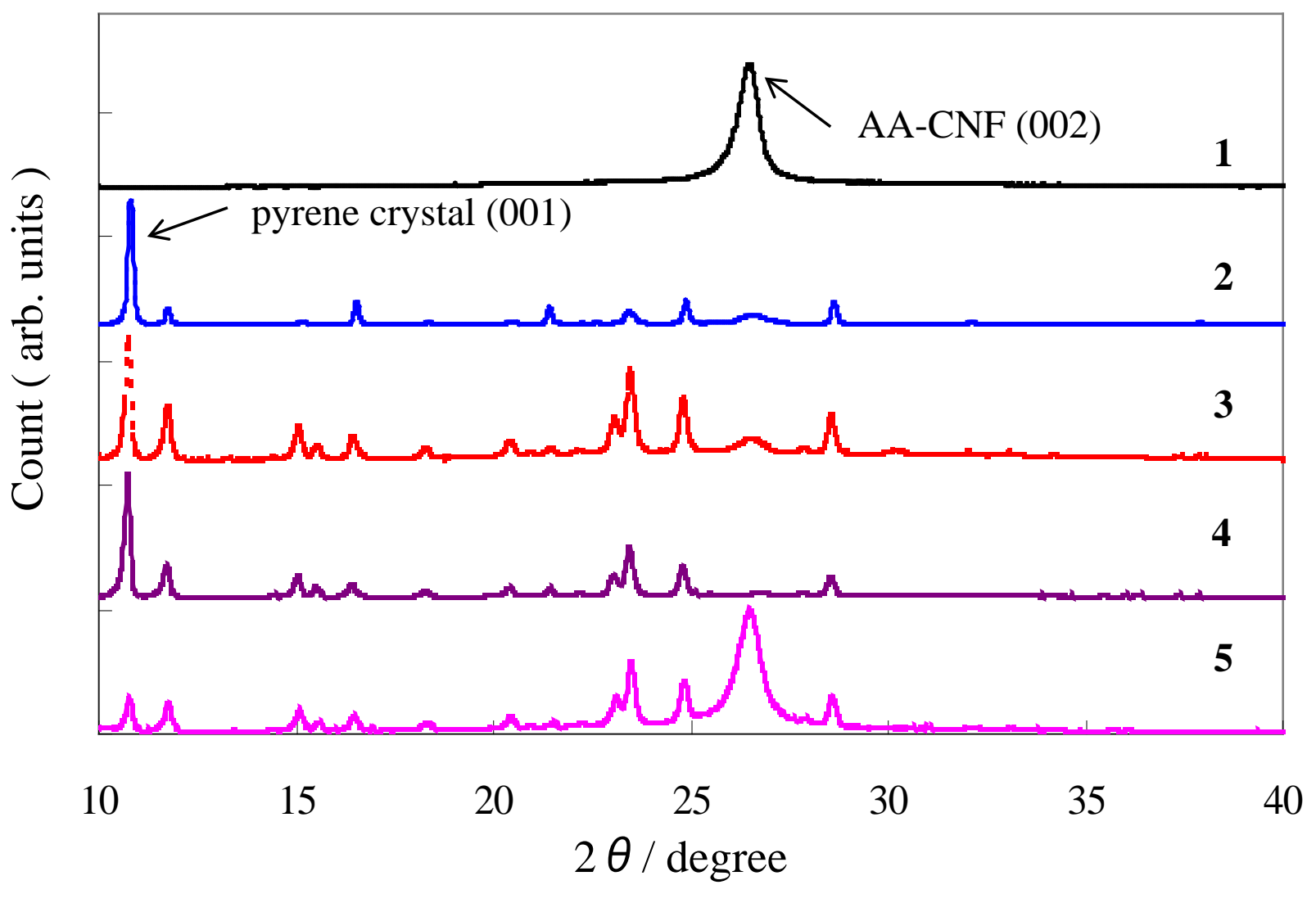


Figure 3

(a)

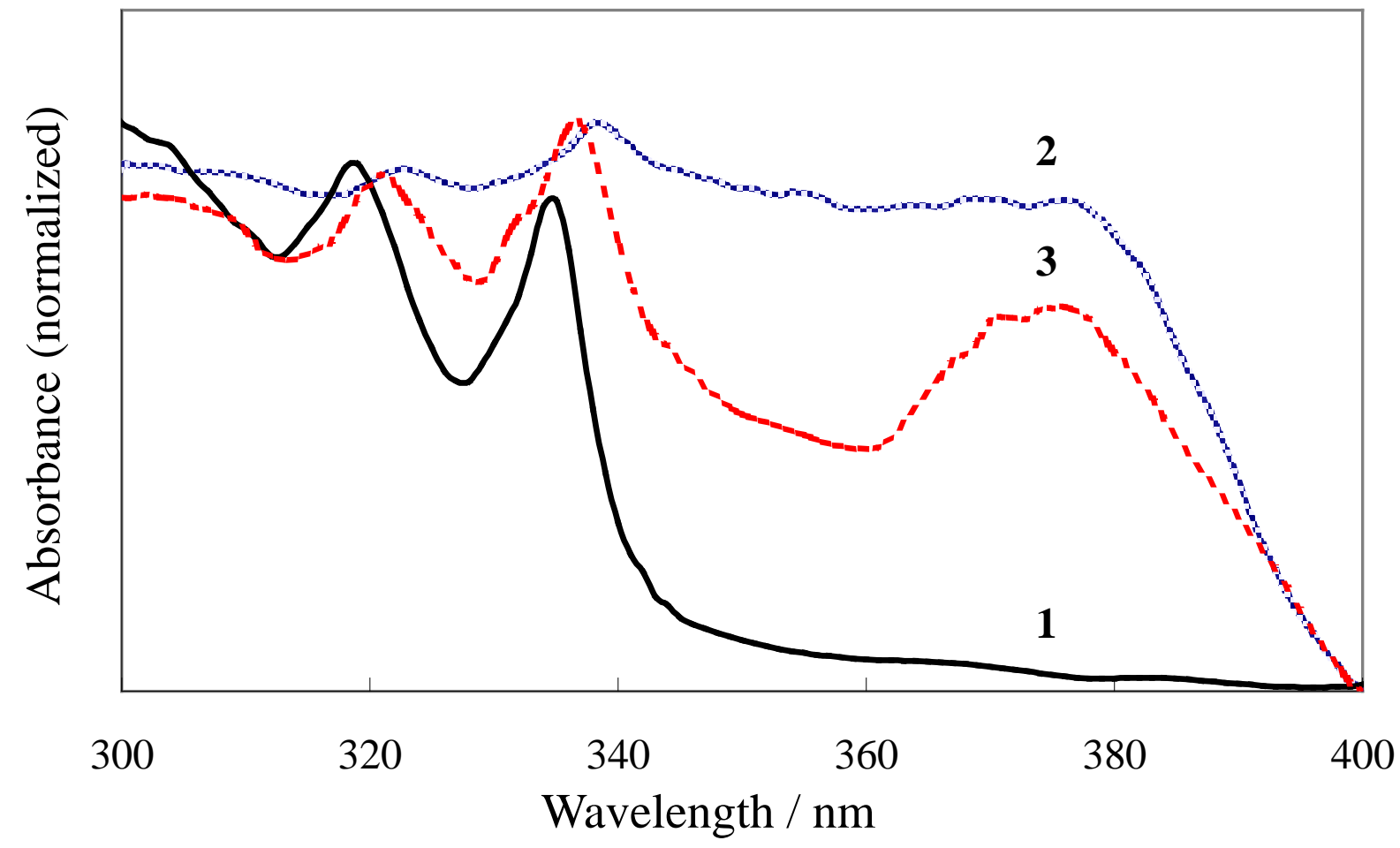

(b)

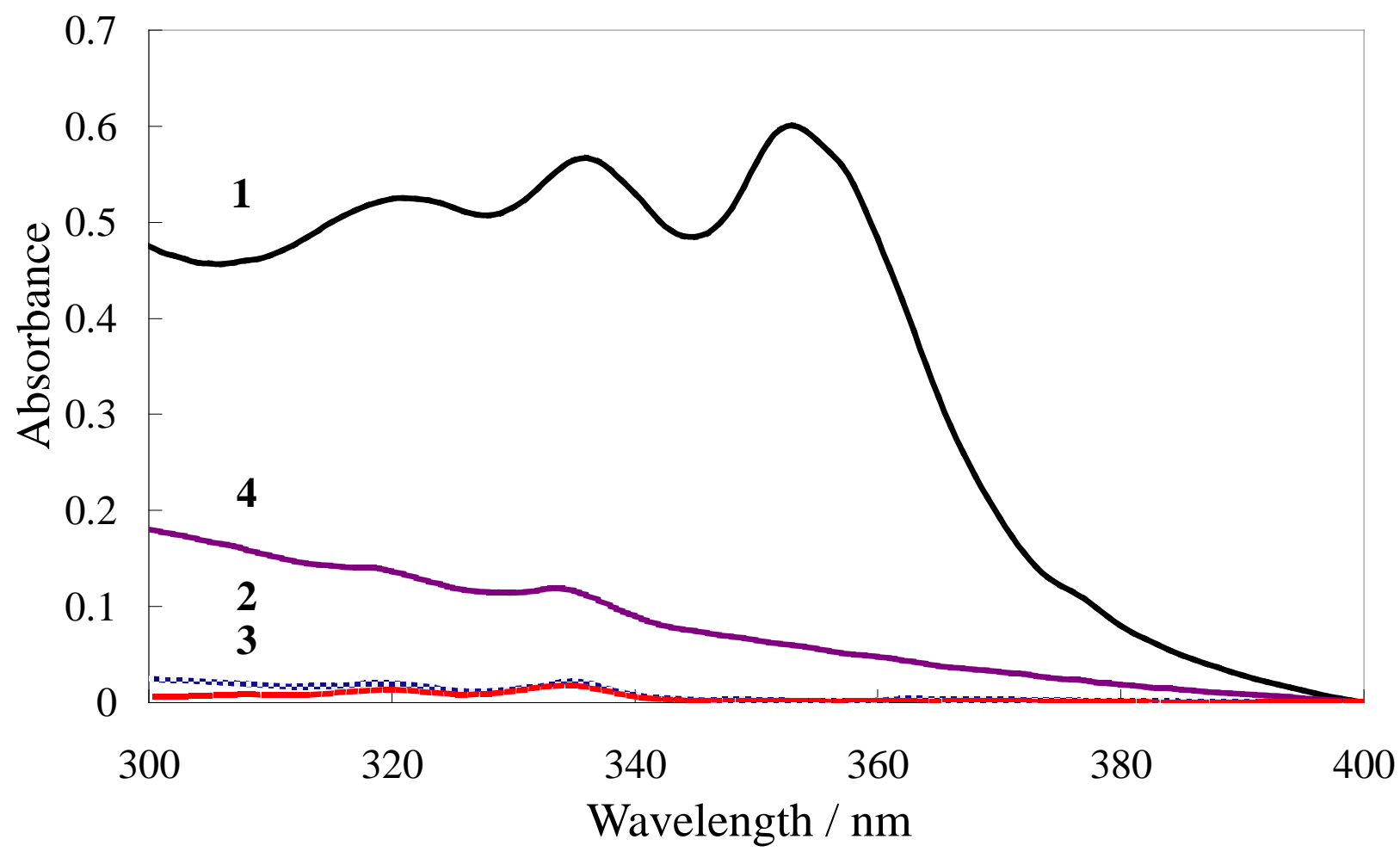


Figure 4

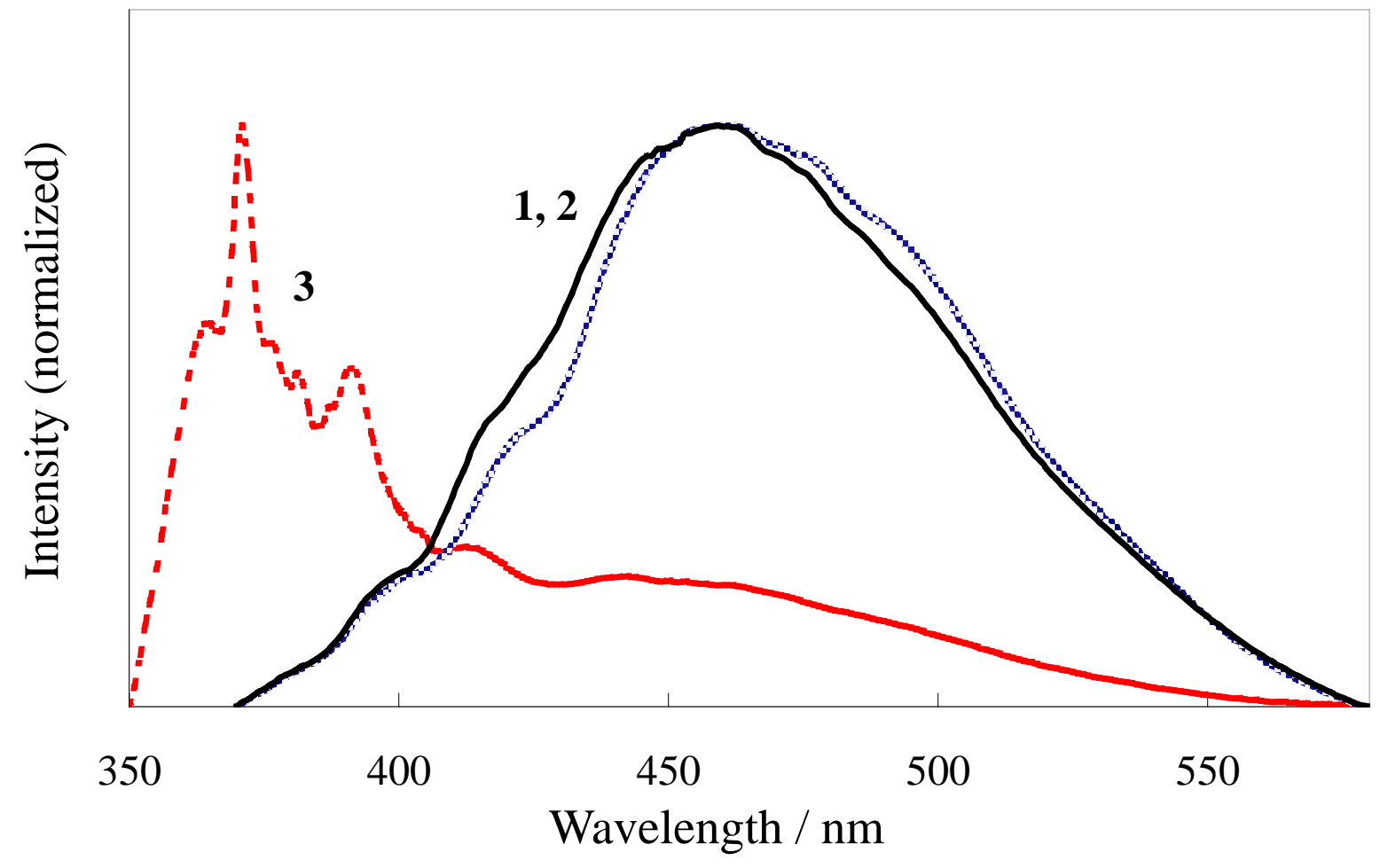


Figure 5

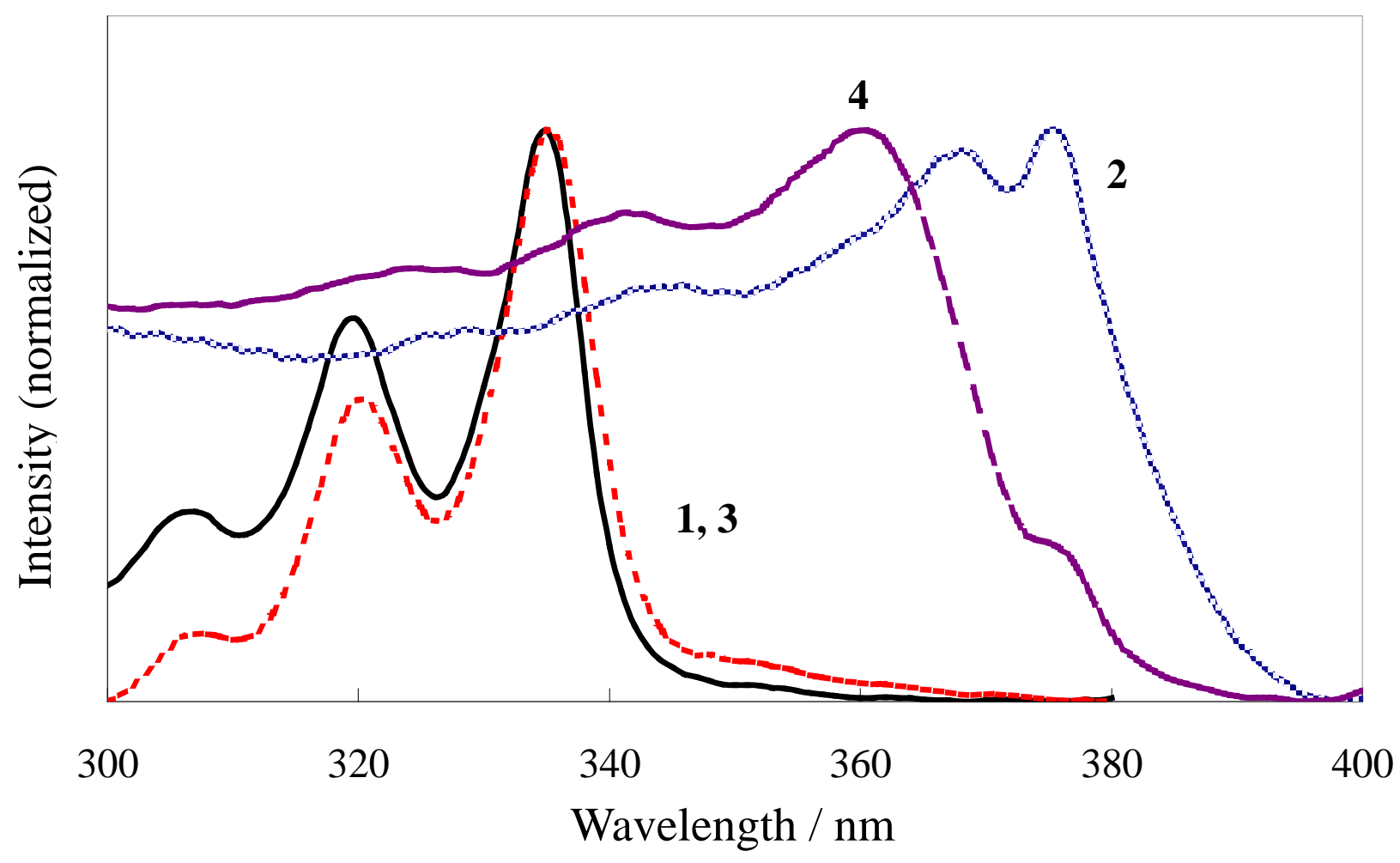

